\title{
Revising autoantibody responses
}

The expression of recombinationactivating gene (RAG) proteins is required for the rearrangement of immunoglobulin genes and receptor editing in immature B cells in the bone marrow. However, the expression of RAG proteins in peripheral B cells is a topic of some controversy. Recent results published in the Journal of Clinical Investigation now show that the re-expression of RAG proteins enables 'receptor revision' in peripheral B cells that are activated by self antigen, thereby preventing autoimmune humoral responses.

The authors of this study made use of an established mouse model in which a lupus-like disease is induced following immunization with a mimetope of double-stranded DNA. Mice immunized with a control peptide that does not result in the generation of autoantibodies served as controls. With this system, the authors clearly demonstrate that
RAG expression was induced in autoreactive B cells, but not in B cells that were specific for the control peptide, following their activation in vivo. Autoreactive $\mathrm{RAG}^{+} \mathrm{B}$ cells were contained within the B-cell fraction that expresses low levels of B220, which contains a large proportion of $\mathrm{CD} 138^{+}$plasma cells, in contrast to the $\mathrm{B} 220^{\mathrm{hi}}$ cell fraction, which is less differentiated. Phenotypic and functional analyses showed that autoreactive B220 low $\mathrm{RAG}^{+} \mathrm{B}$ cells that were derived from germinalcentre responses, rather than extrafollicular responses, had undergone isotype switching and extensive somatic hypermutation, and had features of memory or pre-plasma $\mathrm{B}$ cells. Importantly, further experiments indicated that, although RAGexpressing B cells had differentiated in germinal centres, RAG expression and subsequent receptor revision were induced following activation

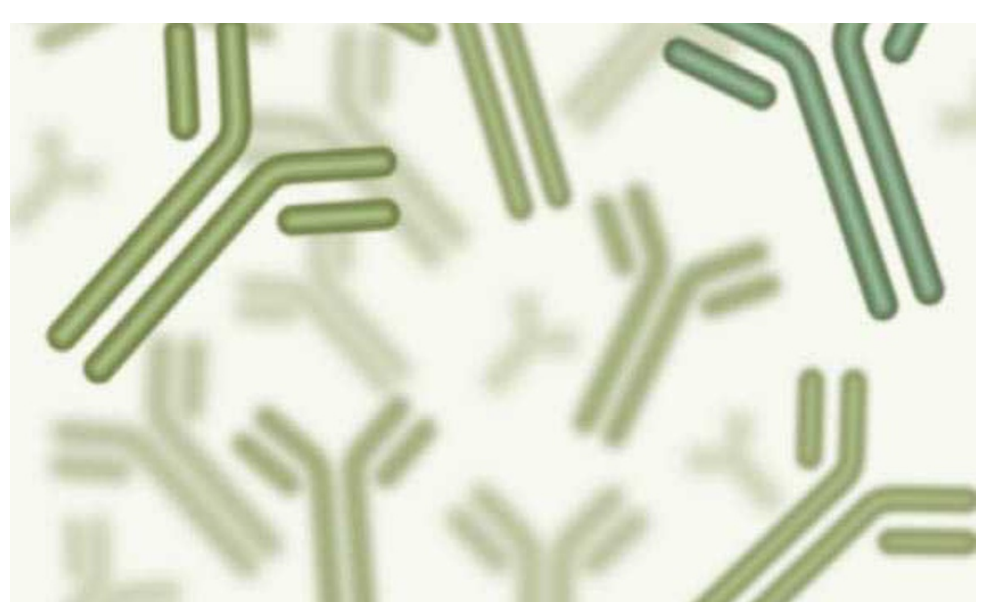

by soluble antigen outside of the germinal-centre environment. If the soluble antigen was eliminated from the circulation - which in this model was achieved by the administration of DNase to remove free self DNA - receptor revision did not occur.

The authors next showed that, similar to the expression of RAG proteins in immature B cells, their re-expression in peripheral $\mathrm{B}$ cells required the expression of interleukin-7 receptor (IL-7R). Blocking of IL-7R inhibited the upregulation of RAG expression by autoreactive $\mathrm{B} 220^{\text {low }} \mathrm{B}$ cells, thereby preventing receptor revision following antigen recognition. The ability to prevent the induction of RAG expression allowed the authors to investigate its importance in limiting autoimmune responses. Indeed, blocking of IL-7R led to a significant increase in the level of serum self-DNA-specific autoantibodies, which indicates that receptor revision is an important mechanism of B-cell tolerance.

Together, these data establish that the expression of RAG proteins following soluble antigen recognition and IL-7R signalling enables receptor revision in autoreactive B cells, which is important for diminishing autoimmune humoral responses.

Sarah Allan

ORIGINAL RESEARCH PAPER Wang, Y. - H. \& Diamond, B. B cell receptor revision diminishes the autoreactive $B$ cell response after antigen activation in mice. J. Clin. Invest. 8, 2896-2907 (2008) 